Rev. Elev. Méd. vét. Pays trop., 1978, 31 (3) : 315-328.

\title{
Impact des lâchers de mâles stériles sur les niveaux de deux populations sauvages de Glossina palpalis gambiensis en Haute-Volta (sources de la Volta Noire)
}

par D. CUISANCE $(*)$, H. POlitZAR $(*)$, M. ClAIR $(*), \operatorname{E} . \operatorname{SELliN}(*)$, Y. TAZE $(*)$

\begin{abstract}
RÉSUMÉ
Des mâles, irradiés à 11000 rads, ont été lâchés pendant 12 mois dans deux gítes expérimentaux constitués par des galeries forestières isolées par des barrières de déboisement, bordant les rivières Gumpy et Guénako, source de la Volta Noire.

Les premiers lâchers ont été effectués en début de saison sèche, 11 et 14 jours après un traitement insecticide non rémanent. Le rapport mâles stériles : mâles sauvages, initialement de $3: 1$ et $7: 1$ sur ces gîtes a atteint des valeurs mensuelles généralement supérieures à $10: 1$.

L'impact des mâles stériles a été apprécié par comparaison avec un secteur témoin, suivant différents critères dont le plus significatif est la forte baisse de densité de $G$. p. gambiensis dans les gîtes expérimentaux.

Le maintien d'une très faible population résiduelle dans les gìtes de lâchers est essentiellement dû à une efficacité insuffisante des barrières qui ont pu être franchies par des glossines venant de l'extérieur ; en effet, le renforcement ultérieur des barrières a permis, en 1978, d'obtenir l'extinction totale de cette population.
\end{abstract}

\section{INTRODUCTION}

Si la lutte classique contre les glossines par pulvérisation insecticide a permis la récupération de vastes zones pâturables dans les régions tropicales à saison sèche bien marquée, la progression actuelle, faisant appel à cette même technique est, sinon compromise, du moins fortement freinée à mesure que l'on pénètre dans les régions à pluviométrie plus forte.

Aussi, de nouvelles méthodes de lutte sont envisagées. La lutte génétique par lâchers de

(*) Centre de Recherches sur les Trypanosomiases Animales B. P. 454, Bobo-Dioulasso, République de Haute-Volta. mâles stériles $(*)$ constitue une de ces voies nouvelles. Elle a fait l'objet d'un certain nombre de travaux de base au laboratoire $(11,13)$. Sur le terrain, deux essais de portée limitée ont laissé entrevoir son utilisation, l'un contre $G$. morsitans morsitans en Rhodésie (7), l'autre contre $G$. tachinoides au Tchad (5). Depuis 1973 et 1975, 2 programmes à grande échelle sont en cours sur le terrain en Tanzanie (U.S. A. I. D.), et en Haute-Volta (I. E. M. V. T.-G. T. Z.).

Cette note a pour intention de donner, après 12 mois de lâcher (décembre 1976-novembre 1977), les premiers résultats concernant l'efficacité de la méthode appliquée à $G$. palpalis gambiensis le long de galeries forestières bordant une partie des sources de la Volta Noire en Haute-Volta.

(*) Le terme de «mâles stériles » est couramment utilisé. Il s'agit ici de mâles soumis à une irradiation qui entraine une stérilité presque totale. 


\section{PROTOCOLE EXPÉRIMENTAL}

\section{Les sites expérimentaux}

\section{a) Description}

Les sites expérimentaux ont été décrits en détail dans une publication précédente (9) ; seules leurs caractéristiques principales sont rappelées ici.

- A $80 \mathrm{~km}$ de Bobo-Dioulasso, la région d'Orodara constitue un véritable «chateau d'eau " d'où 2 rivières prennent leurs sources, la Guénako et la Guimpy, qui se joignent en aval pour donner la Volta Noire.

- Ces rivières permanentes sont bordées de galeries forestières homogènes, continues, larges de 5 à $20 \mathrm{~m}$, constituées de 3 espèces arborées dominantes (Berlinia grandiflora, Carapa procera, Uapaca somon), dont la canopée forme une voûte jointive au-dessus du lit, généralement très encaissé (schéma $\mathrm{n}^{\circ} 1$ ).

- Par suite de la disparition de la faune sauvage, les villageois et leur bétail sont les hôtes nourriciers essentiels de 2 espèces de glossines, Glossina palpalis gambiensis, bien représentée (95 p. 100) et Glossina tachinoides, plus rare (5 p. 100).

- Trois tronçons de galerie forestière, longs chacun de $5,5 \mathrm{~km}$ ont été délimités :

- un gîte témoin, situé sur la Guénako (amont du village Kourinion) ;

- un gîte de lâcher, situé sur la Guénako (aval du village Kourinion) ;

- un second gîte de lâcher, situé sur la Guimpy ;

- On a cherché un isolement maximal de ces
3 gîtes en choisissant la partie amont de ces rivières et en créant des barrières d'éclaircissement total (déforestation totale et traitement arboricide des souches) renforcées d'une part, par des pulvérisations bimensuelles d'insecticides rémanents (D. D. T., Dieldrin) et d'autre part, par la mise en place, tous les $100 \mathrm{~m}$, de pièges biconiques contrôlés chaque semaine. Les longueurs des barrières varient, selon leur situation plus ou moins stratégique, de $0,300 \mathrm{~km}$ (barrières $\mathrm{C}$ et $\mathrm{D}$ ) à $1,300 \mathrm{~km}$ (barrières A et B) (cf. carte $n^{\circ} 1$ );

- un réseau de $30 \mathrm{~km}$ de pistes longe au plus près le lit des cours d'eau afin de desservir les points de lâchers et de sondages, ainsi que les barrières pour leur entretien.

\section{b) Evaluation des populations de glossines}

Des sondages effectués, par captures-marquages-recaptures, en novembre 1976, en début de saison sèche, avant les lâchers, indiquent les densités réelles suivantes, pour G.p. gambiensis :

- Témoin : 1200 glossines.

- Guénako : 1800 glossines.

- Guimpy : 600 glossines.

\section{Traitement insecticide non rémanent}

- L'Endosulfan, insecticide choisi, a été épandu à la concentration de 5 p. 100 à l'aide d'appareils «Swingfog» qui projettent un brouillard de gouttelettes dont la taille très faible n'entraîne pratiquement aucune rémanence.

D'après les sondages de contrôle effectués les jours suivants, la durée de la rémanence est estimée vraisemblablement par excès à environ 5 à 7 jours.

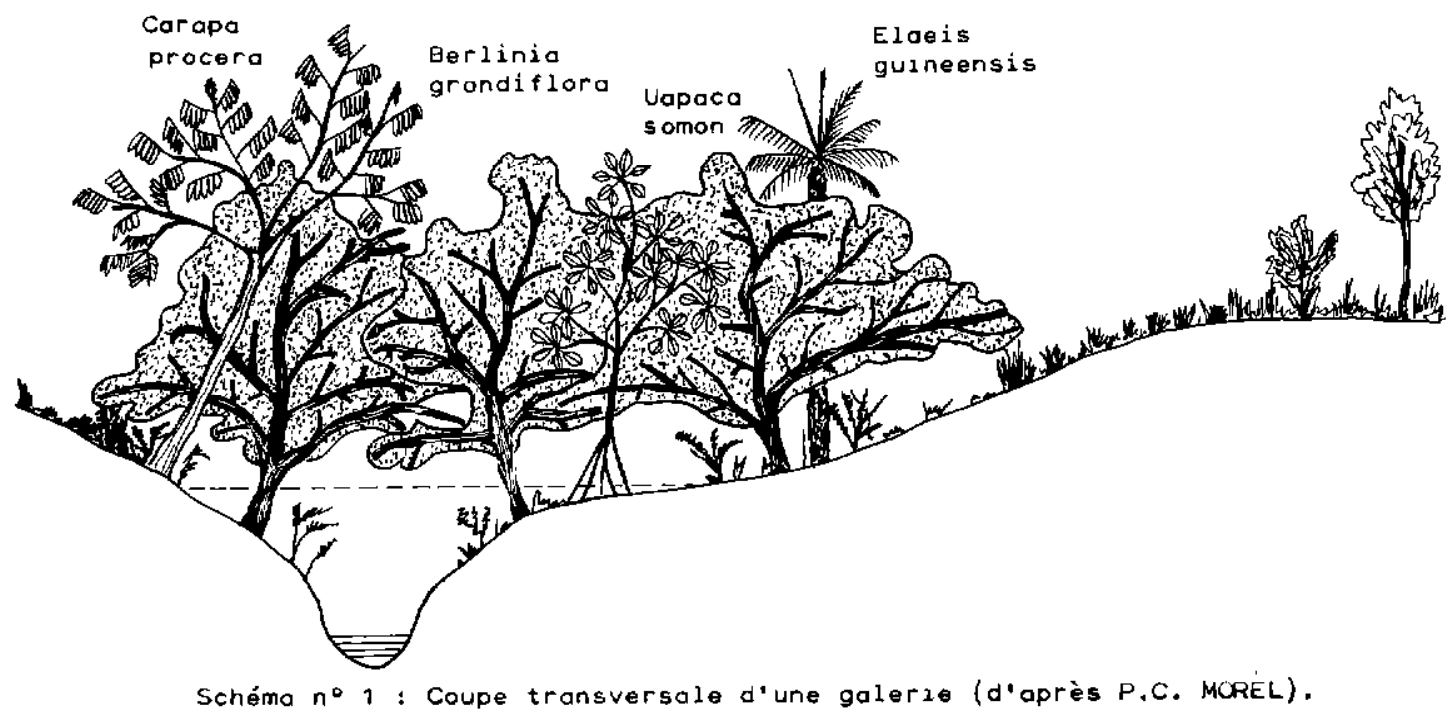


Carte $n^{0}$ 1-Gîte d'expérimentation

de la méthode du mâle stérile contre

G. palpalis gambiensis.

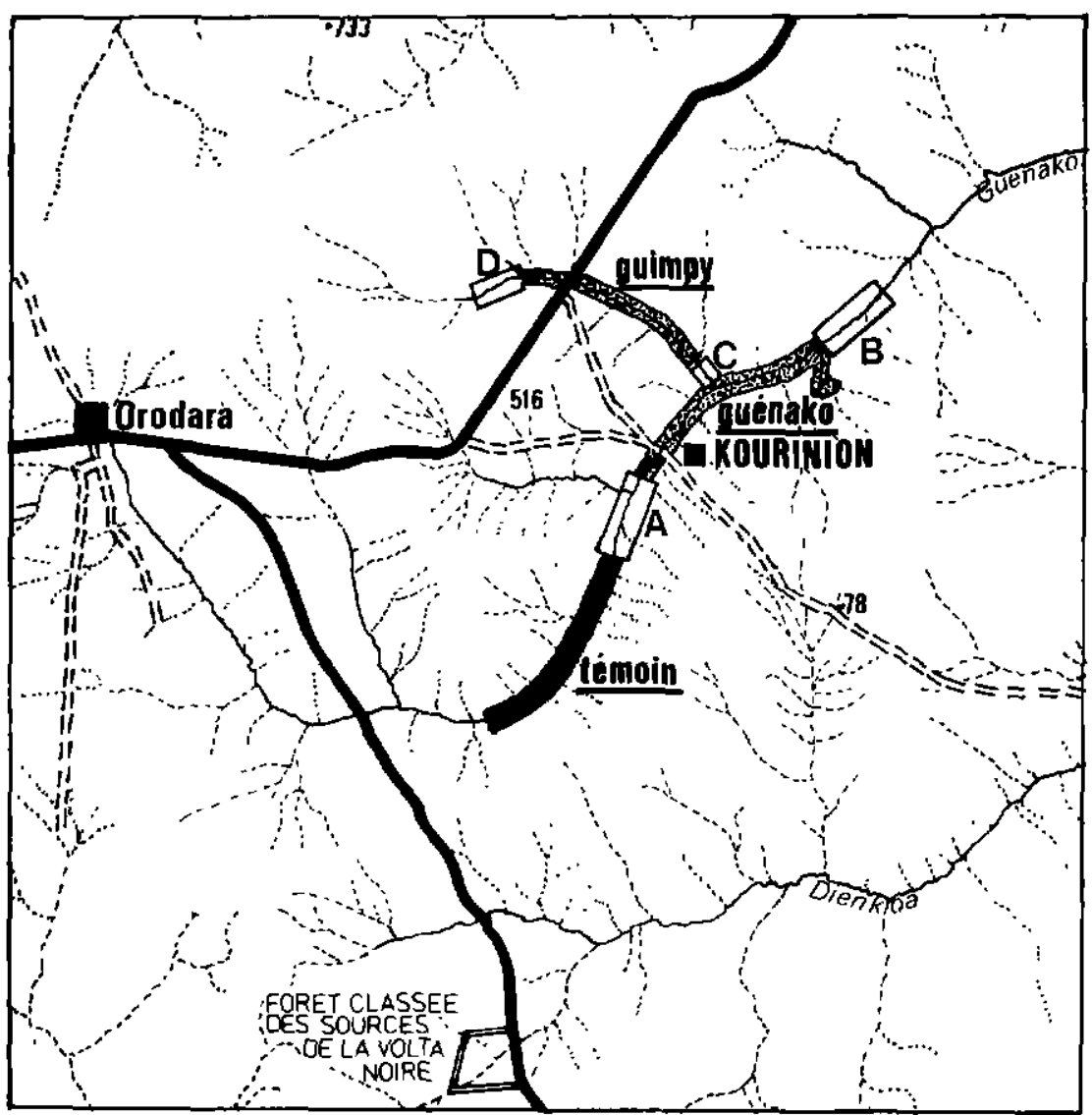

Eche11e au

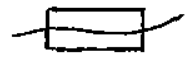

barrière

$1 / 200$ oope

tronçons expërimentaux

tronçon témoin

- Du traitement insecticide non rémanent, on espère 2 effets :

- un abaissement de la densité naturelle, destiné à obtenir un rapport favorable entre mâles irradiés et mâles sauvages.

L'insecticide élimine la population adulte sauvage, présente le jour du traitement, sans porter atteinte au stock de pupes enfouies dans le sol, à partir duquel elle ya se reconstituer.

La dynamique prévisionnelle de cette reconstitution (schémas $n^{\text {os }} 2$ et 3 ) est établie, en tenant compte des caractéristiques biologiques de l'espèce, telles que déterminées par nos travaux antérieurs, à partir des effectifs avant traitement ;
- un rajeunissement de la population par élimination des adultes de sorte que seules les jeunes femelles qui éclosent se trouvent en présence des mâles irradiés lâchés.

\section{Lâchers de mâles irradiés}

En se basant d'une part sur les densités observées après le traitement insecticide non rémanent, d'autre part sur le rendement de la colonie en élevage, on a adopté un protocole de lâcher assurant aux mâles stériles les meilleures conditions possibles de compétitivité.

\section{- Rapport mâles stériles: mâles sauvages}

Compte tenu de la table de survie établie en 


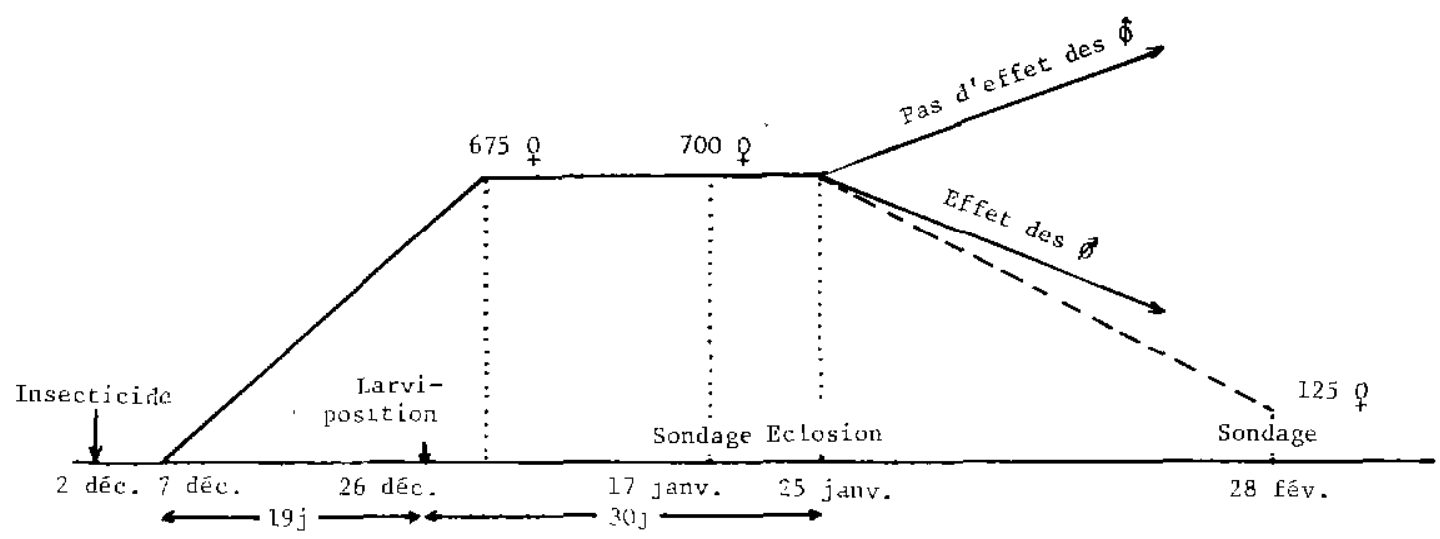

Schéma $n^{\bullet} 3$ - Dynamique prévisionnelle de la densitë de population sur Guimpy.

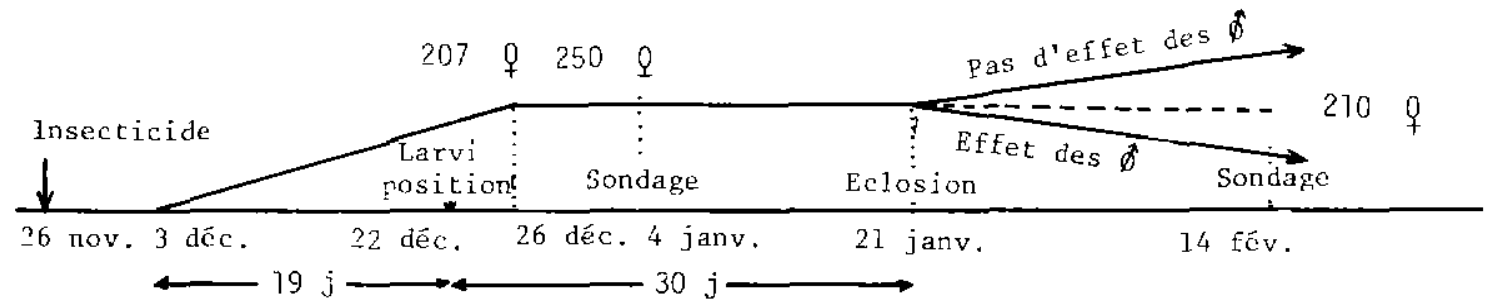

1976 pour les mâles stériles (4), on a modulé les quantités lâchées durant les 8 premiers jours afin d'obtenir les taux initiaux suivants :

- sur Guénako: 3 mâles stériles pour 1 mâle sauvage,

— sur Guimpy : 7 mâles stériles pour 1 mâle sauvage.

Les quantités lâchées ensuite sont maintenues constantes.

Il y aura donc légère baisse de la valeur des rapports prévus jusqu'à la fin de l'éclosion des adultes issus des pupes enfouies dans le sol, puisque les quantités lâchées demeurent stables, alors que des adultes éclosent. Passé ce délai, il y aura au contraire élévation de la valeur de ces rapports, puisqu'il n'y aura plus aucune éclosion d'adultes et que la mortalité naturelle interviendra alors.

\section{- Intervalle des lâchers dans le temps et dans} l'espace

On lâche les mâles stériles à raison de 2 séances par semaine, le mardi et le vendredi sur Guénako et Guimpy.

Les quantités lâchées sont fractionnées en 28 points espacés de $200 \mathrm{~m}$ chacun.

\section{-- Epoque des lâchers}

Les lâchers commencent en saison sèche, période jugée la plus propice pour atteindre l'espèce-cible regroupée sur une faible surface : G. p. gambiensis reste en effet fortement inféodée aux galeries forestières qu'elle ne quitte pratiquement pas à cette époque.

La dynamique de population pour cette espèce et pour cette zone climatique indique que les densités sont encore hautes en début de saison sèche (novembre-décembre) mais qu'elles vont en s'abaissant jusqu'au mois de mai (9).

Les lâchers ont commencé au mois de décembre. Les contingences de la production et du transport ont imposé l'après-midi comme moment des lâchers.

\section{- Etat de l'insecte lâché.}

Les insectes irradiés ont été lâchés à l'état adulte plutôt qu'à l'état pupal, car les lâchers d'adultes :

- ne nécessitent pas une irradiation et un transport sous azote et sous froid ;

- permettent la libération d'individus qui ont déjà pris un repas de sang, donc qui survivront mieux; 
- atténuent fortement le risque de vecteur potentiel des mâles stériles qui auront pris leur premier repas de sang sur un lapin sain ce premier repas conditionnant en grande partie chez la glossine son pouvoir infectant futur ;

- évitent une trop grande perte par prédation dans la nature du fait du pouvoir de dispersion des imagos, ce qui n'est pas le cas des pupes immobiles, donc vulnérables ;

- facilitent le marquage des individus, celui-ci demeurant visible sur l'adulte vivant.

Les mâles sont irradiés à la dose de 11000 rads fournie par le rayonnement gamma d'une source de Caesium 137 (12 400 curies). Cette dose est un compromis entre une stérilité subtotale de 95 p. 100 et une longévité très acceptable au laboratoire (16).

\section{- Transport.}

Après divers essais, un caisson simple, solide et très efficace a été construit localement : sorte de cage à mailles métalliques larges tapissée sur toute sa surface par un matelassage humidifié (toile de jute et coton) et s'ouvrant par sa partie supérieure. Il peut contenir 900 mâles stériles répartis dans 36 cages Roubaud.

\section{Contrôle de l'expérimentation}

L'objectif est d'apprécier l'impact des lâchers de mâles stériles sur la population sauvage, donc de comparer l'évolution des densités de population sur les gîtes d'expérience (GuénakoGuimpy) par rapport au gîte témoin.

\section{Moyens}

Les sondages se font par capture à l'aide de 28 pièges biconiques CHALLIER-LAVEISSIERE (1), disposés tous les $200 \mathrm{~m}$ au bord de l'eau, le long des $5,5 \mathrm{~km}$ de galerie forestière de chaque gite.

\section{Méthodologie}

- Les pièges sont posés à $9 \mathrm{~h}$ par 2 équipes, chacune mettant en place 14 pièges. La durée de piégeage est de 5 h $1 / 2$, le retrait ayant lieu à $14 \mathrm{~h} 30$.

En saison sèche, une récolte intermédiaire a lieu à midi du fait des rigueurs climatiques, néfastes aux glossines.

Transportées en caisson humidifié, les glossines sont immobilisées par le froid, les cages étant prises entre 2 barres de glace puis elles sont triées.
Tous les mâles stériles produits au laboratoire sont porteurs d'une marque de gouache acrylique.

L'estimation de la densité réelle des glossines sauvages s'effectue par capture, marquage et recapture (indice de Lincoln simple) à $48 \mathrm{~h}$ d'intervalle. Afin de perturber au minimum les gîtes, une estimation a lieu tous les 42 jours sur chaque gîte. Les couleurs de marquage des glossines sauvages sont différentes de celles des mâles stériles.

L'estimation de la densité apparente s'exprime en nombre de glossines prises en une séance de capture par les 28 pièges.

La « disponibilité » (ou « availability»), qu est le rapport densité réelle/densité apparente, a été calculée sur 2 années d'observation. Il est en moyenne voisin de 10 sur les 3 gîtes : la capture d'une $G$. palpalis gambiensis correspond à la présence de 10 individus dans le gîte.

\section{RÉSULTATS}

\section{Lâchers}

\section{a) Quantités lâchées}

Sur 155000 mâles stériles produits, 69758 se sont envolés sur Guénako et 66401 sur Guimpy soit un pourcentage d'envol de 87,71 p. 100 et 88,19 p. 100 .

Les pertes globales représentées par les glossines mortes et par celles qui n'ont pu s'envoler (malformations, faiblesse, traumatismes, etc.) sont en moyenne de 12,28 p. 100 pour Guénako et 11,81 p. 100 pour Guimpy et sont comparables $\left(X^{2}=0,04\right.$; d. d. I. $=1$; N. S. $)$;

Le taux résulte, d'une part de pertes au niveau de l'élevage (entre le moment de l'éclosion et celui de l'irradiation des mâles), d'autre part de pertes entre la sortie du laboratoire et le moment du lâcher (transport).

Ces données, très acceptables, sont voisines de celles obtenues 1 an auparavant sur les mêmes gites (9), ce qui tend à montrer qu'en zone tropicale soudanienne il est difficile de descendre en dessous d'un taux moyen de pertes de 12 p. 100, dû pour une part au maintien difficile de la constance climatique dans les insectariums (pannes d'eau, d'électricité, de machines, fautes professionnelles, écarts climatiques saisonniers importants etc), ainsi qu'aux écarts bioclimatiques entre le lieu d'élevage et le lieu de lâcher. 


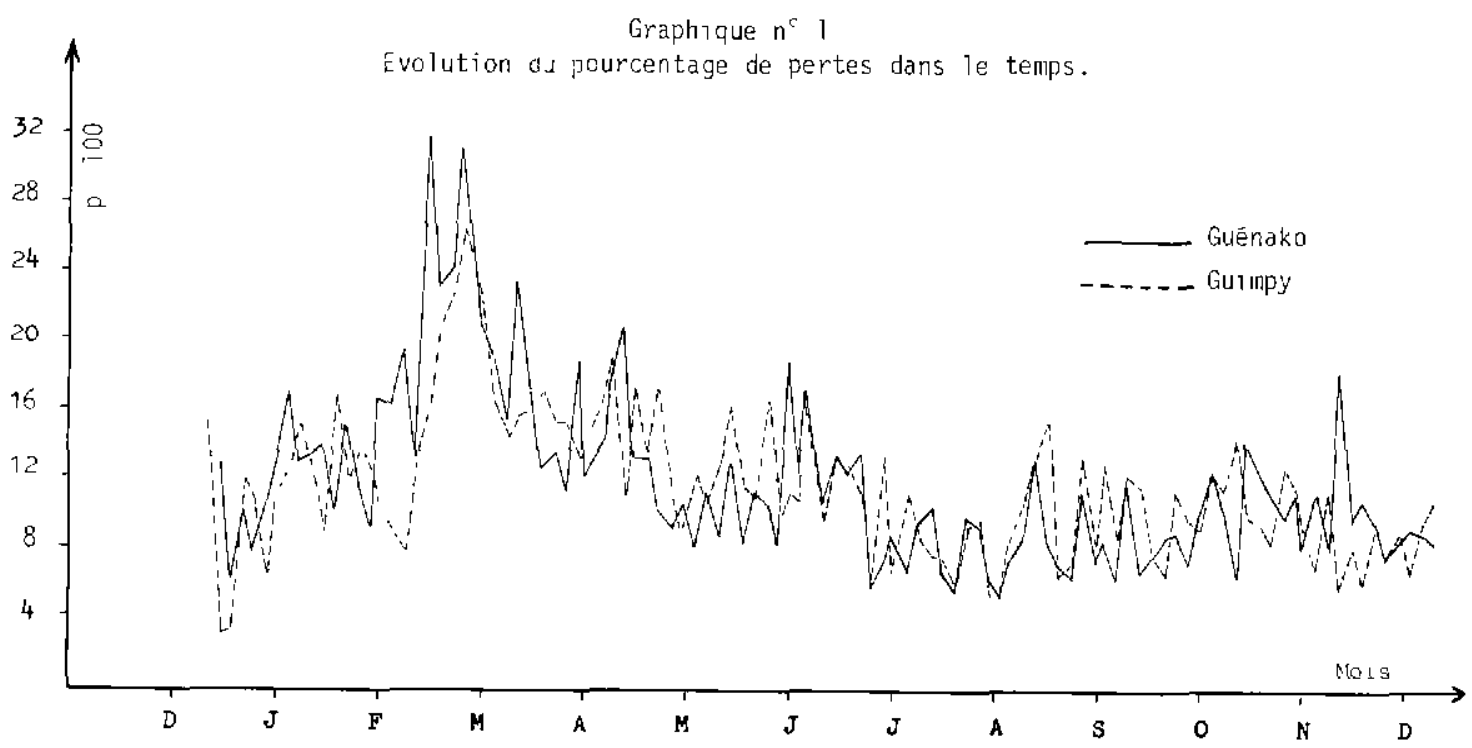

En effet, l'évolution du taux de perte au cours de l'année (graphique $n^{\circ} 1$ ) indique un pic assez fort en février-mars, attribuable au fonctionnement défectueux d'un humidificateur dans un insectarium. On remarque cependant que, jus. qu'à mai-juin, la courbe est au-dessus de 12 p. 100 tandis qu'elle est au-dessous ensuite.

C'est donc en saison sèche que les plus grandes pertes sont enregistrées. Cclles-ci baissent lorsque les lâchers s'effectuent en saison à hygrométrie moyenne ou haute, ce qui montre le rôle néfaste des variations brutales de climat entre les salles d'élevage et la galerie forestière, lors des lâchers en saison sèche. La transition inverse aboutit aux mêmes résultats : des glossines récoltées dans la nature en saison sèche et rentrées au laboratoire meurent en fortes proportions.

\section{b) Points et fréquences de lâchers}

A chaque séance, le fractionnement des quantités lâchées en 28 points espacés de $200 \mathrm{~m}$ tant sur Guénako que sur Guimpy a été rigoureusement respecté.

La production régulière de l'élevage a permis d'appliquer le rythme de 2 lâchers par semaine sur chaque tronçon expérimental soit :

- 100 lâchers sur Guénako avec 1400 mâles stériles par semaine (prévisions : 1400 mâles stériles par semaine);

- 101 lâchers sur Guimpy avec 1300 mâles stériles par semaine (prévisions: 900 mâles stériles par semaine).

TABLEAU $\mathrm{N}^{\circ} \perp$

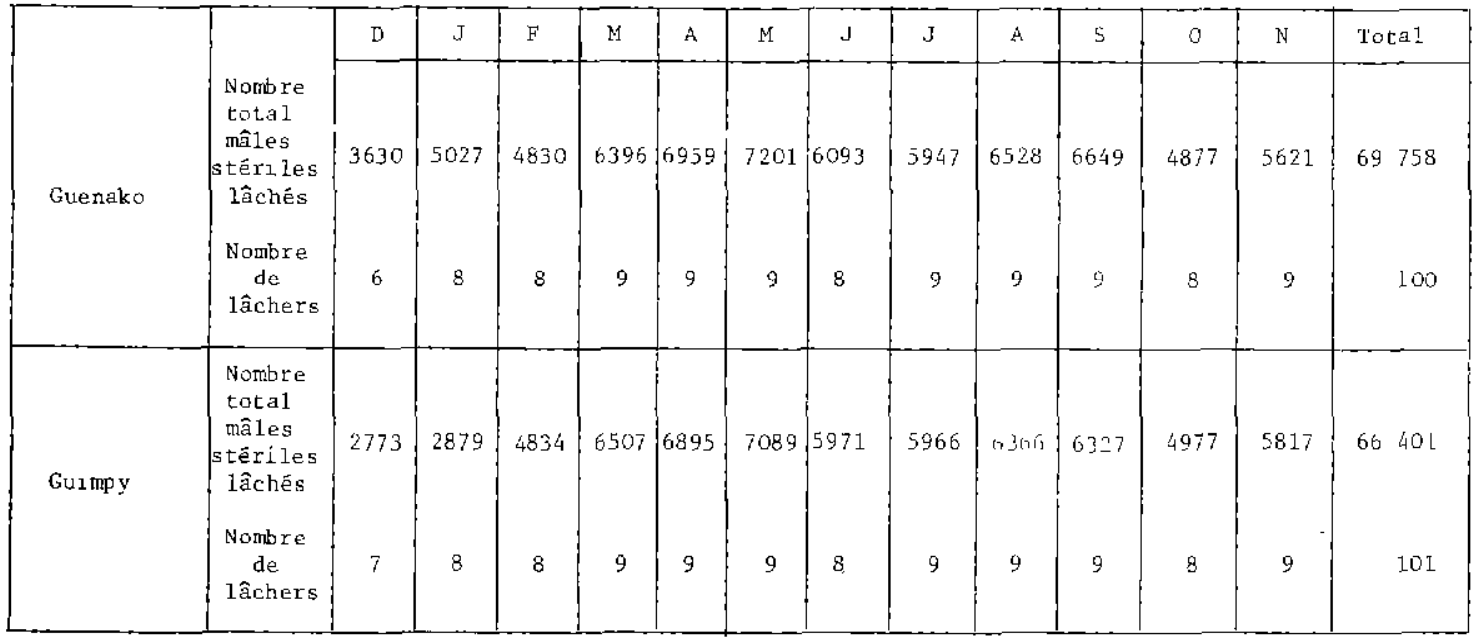


Les quantités prévues ont donc été respectées sur Guénako et majorées sur Guimpy.

\section{c) Rapports mâles stériles/mâles sauvages dans les gites}

Les sondages effectués tous les 42 jours sur chacun des gites (chaque sondage comprend 2 captures à $48 \mathrm{~h}$ d'intervalle) permettent de comptabiliser facilement les mâles stériles et les mâles sauvages pris dans les pièges et d'avoir une bonne idée du rapport de nombre existant entre eux au cours de l'année, puisqu'ils se distinguent par le système de marquage. On peut ainsi suivre l'évolution chronologique des niveaux de population des mouches sauvages et introduites.

TABL. $N^{\circ} I I-R a p p o r t ~ \phi / 0^{*}$ d'après les résultats des captures aux pièges.

\begin{tabular}{|l|c|c|}
\hline \multirow{2}{*}{ Décembre } & Guénako & Guimpy \\
\cline { 2 - 3 } & $2,57: 1$ & $8,00: 1$ \\
\hline Janvier & $2,35: 1$ & $1,89: 1$ \\
\hline Février & - & $5,03: 1$ \\
\hline Mars & $18,05: 1$ & $7,67: 1$ \\
\hline Avril & $7,79: 1$ & - \\
\hline Mai & $22,56: 1$ & $19,06: 1$ \\
\hline Juin & $18,17: 1$ & $15,78: 1$ \\
\hline Juillet & $42,33: 1$ & - \\
\hline Août & $17,92: 1$ & $18,50: 1$ \\
\hline Septembre & $39,83: 1$ & $13,27: 1$ \\
\hline Octobre & - & $33,92: 1$ \\
\hline Novembre & $19,18: 1$ & - \\
\hline
\end{tabular}

Durant les 12 mois d'observation, sur 2022 captures, le taux moyen théorique est de presque 10 mâles stériles pour 1 mâle sauvage sur les 2 gîtes.

- 9,33 mâles stériles pour 1 mâle sauvage sur Guénako (2100 Ø̋: 225 ठే) ;

- 9,81 mâles stériles pour 1 mâle sauvage sur Guimpy (3 $504 \varnothing$ : 357 \%).

En pratique, ainsi que le tableau $n^{\circ}$ II l'indique, les taux de mâles stériles ont été souvent supérieurs à $10: 1$.

Les sondages effectués dans la première semaine suivant le premier lâcher montrent que les taux prévisionnels sont respectés :
- 2,57 mâles stériles pour 1 mâle sauvage sur Guénako (prévu : $3: 1$ ) ;

- 8,00 mâles stériles pour 1 mâle sauvage sur Guimpy (prévu : $7: 1$ ).

Dans le mois suivant, il y a baisse de ce rapport puisque les quantités lâchées demeurent constantes et que les imagos sauvages éclosent à partir du stock de pupes déposées dans le sol des gîtes.

A partir de février, le rapport remonte selon les prévisions, mais de façon importante, car la bonne production de l'élevage a permis des lâchers excédentaires. Ils ont oscillé entre 5000 et 7000 mâles stériles par mois sur chaque gîte et sont demeurés quantitativement stables.

On constate alors que ce rapport tend à s'élever pour atteindre des valeurs extrêmes de haut niveau entre juillet et octobre :

- 42,33 $\varnothing: 1$ ô sur Guénako;

- 33,92 ø: 1 ô sur Guimpy.

L'élévation dans le temps du rapport $\varnothing$ : $\sigma^{\star}$, alors que les quantités de mâles stériles lâchés ne s'accroissent plus, ne peut être que la conséquence d'une diminution progressive de la densité de la population de mâles sauvages.

Le fait que les résultats observés pour les 2 gites soient comparables peut surprendre, du moment que les effectifs sauvages étaient au départ très différents. Le contrôle des barrières et l'usage de systèmes de marquage a montré que la barrière $\mathrm{C}$ a permis des passages de glossines de Guimpy vers Guénako alors qu'inversement des mâles sauvages ont pu passer à travers la barrière $D$ vers la Guimpy, ce qui a eu pour effet de rendre les résultats définitifs plus homogènes.

\section{Effets des lâchers sur la population sauvage.}

Les méthodes d'évaluation de l'efficacité de la lutte génétique ne sont actuellement pas codifiées. Il convient donc de trouver les critères permettant de mesurer au mieux l'impact des mâles stériles sur une population sauvage. On a retenu les suivants :

- évolution du sex-ratio de G.p. gambiensis,

- évolution relative des densités de population de G. p. gambiensis et de G. tachinoides,

- évolution de la densité de population de G. p. gambiensis (comparaison entre les gîtes),

- évolution de la fertilité des femelles sauvages mises en observation. 
a) Evolution du sex-ratio de G. p. gambiensis

Les mâles sont irradiés à une dose provoquant une stérilité de 90-93 p. 100 . On peut penser que l'accouplement des mâles avec des femelles sauvages provoquera des perturbations du rapport des sexes dans la descendance réduite de ces dernières, comme cela a été démontré au laboratoire $(6 ; 12)$.

L'étude du sex-ratio ( $(\hat{\delta}:$ o ) dans la nature devrait constituer alors un indice de l'effet des mâles stériles. Or une telle appréciation est difficile car l'attractivité du piège biconique vis-à-vis des 2 sexes est certainement différente chez G. p. gambiensis. Elle varie de plus avec les saisons et avec l'emplacement du piège (1).

On constate toutefois que, sur les 3 tronçons expérimentaux, le sex-ratio a une valeur moyenne qui demeure toujours supérieure à 1 , donc nettement en faveur des mâles.

\begin{tabular}{|c|c|c|c|}
\hline$M \circ i s$ & Guénako & Guimpy & Témoin \\
\hline Dëcembre & - & - & $1,59: 1$ \\
\hline Janvier & $1,23: 1$ & $1,97: 1$ & - \\
\hline Fëvrier & $0,50: 1$ & $1,20: 1$ & $0,95: 1$ \\
\hline Mars & - & $0,94: 1$ & $1,36: 1$ \\
\hline Avril & $1,36: 1$ & - & $1,20: 1$ \\
\hline Mai & $8,00: 1$ & $2,25: 1$ & - \\
\hline Juin & $2,00: 1$ & $3,83: 1$ & $3,27: 1$ \\
\hline Juillet & $3,00: 1$ & - & $2,52: 1$ \\
\hline Août & $2,40: 1$ & $6,00: 1$ & $4,00: 1$ \\
\hline Septembre & $0,67: 1$ & $5,00: 1$ & $3,75: 1$ \\
\hline Octobre & - & $2,00: 1$ & $3,4 \overline{7}: 1$ \\
\hline Novemb re & $0,73: 1$ & - & $2,01: 1$ \\
\hline Moyenne $=$ & $1,23: 1$ & $1,49: 1$ & $1,55: 1$ \\
\hline
\end{tabular}

Dans les captures de glossines sauvages, les mâles dominent significativement les femelles sur Guénako (207 mâles/168 femelles; $X^{2}=4,08$; d. d. 1. = 1), sur Guimpy (349 mâles/235 femelles ; $X^{2}=22,25 ;$ d. d. $\left.1 .=1\right)$ et sur le témoin (1 100 mâles $/ 708$ femelles; $X^{2}=84,98 ;$ d. d. $1 .=1$ ).

Toutefois, il n'y a pas de différence significative entre les sex-ratio enregistrés sur les 3 gîtes :
Guénako-Guimpy: $t=0,64 ;$ N. S. ; d. d. $1 .=15$. Guénako-Témoin : $t=0,24 ; \mathrm{N}$. S. ; d.d. $1 .=17$. Guimpy-Témoin : $\mathrm{t}=0,68 ; \mathrm{N}$. S. ; d. d. $1 .=16$.

Il est donc difficile pour le moment de se faire une idée sur la valeur de ce critère. Cependant, des accouplements mâles irradiés $\times$ femelles non irradiées au laboratoire (non publié) montrent en $\mathrm{F} 1$ une déviation du sex-ratio en faveur des mâles, constatation également faite par ITARD (12) chez G. tachinoides, CURTIS et collab. (6) chez G. morsitans morsitans, TAZÉ et Collab., (16) et ITARD (11) chez G. palpalis gambiensis, EVENS et Collab., (8) chez G. palpalis palpalis.

b) Evolution relative des densités de population de G. p. gambiensis et de G. tachinoides

- Sur l'ensemble de l'aire expérimentale, le piège biconique attrape en moyenne 94,30 p. 100 de G. p. gambiensis et seulement 5,70 p. 100 de $G$. tachinoides, avec une fréquence décroissante de G. tachinoides :

$$
\text { Guénako > Témoin > Guimpy. }
$$

Sur le gîte témoin, après un pic en février, on assiste à une baisse du rapport G.p. gambiensis/ $G$. tachinoides durant la saison sèche, suivie d'une remontée en saison des pluies, due à l'élévation de densité de G. p. gambiensis selon l'évolution très classique observée normalement dans cette zone climatique, chez cette espèce.

Par contre, sur les 2 gîtes Guénako et Guimpy, on note, après le pic de février, une chute constante de la valeur de ce rapport, ce qui traduit une baisse de fréquence de $G$. p. gambiensis relativement à $G$. tachinoides, considérée comme espèce de référence.

Donc, sur les 2 gîtes receveurs de mâles stériles, la densité de G.p. gambiensis a baissé constamment par rapport à celle de $G$. tachinoides et ne s'est pas élevée après la saison humide comme sur le témoin.

Cette baisse du rapport G. palpalis/G. tachinoides est en fait sous-estimée. En effet, ces gîtes sont séparés par des barrières renforcées ellesmêmes par des séries de pièges biconiques, or on sait (1) que ces pièges prennent davantage G. tachinoides que G. p. gambiensis, en particulier en zone découverte.

La densité de $G$. tachinoides a donc subi une baisse due à l'effet surajouté des barrières, qui est évalué à presque 2 fois celui exercé sur G. $p$. gambiensis. 
TABL. $N^{\circ} I V$-Quantitës respectives des deux espèces capturées sur les trois gites pendant l'année.

\begin{tabular}{|c|c|c|c|}
\hline & G. $p \cdot$ gamiiensis & a. tachincides & a. plipatio/s. tamilinotates \\
\hline Guérako & $375(89,50 \mathrm{p} .100)$ & $44(10,50$ p. 100$)$ & $8,52: 1$ \\
\hline Guimpy & $584(98,30 \mathrm{p} .100)$ & $10 \quad(1,70 \mathrm{p}, 100)$ & $58,40: 1$ \\
\hline Témoin & $1808(94,20$ p. 100$)$ & $112(5, B 0 p \cdot 100)$ & $16,14: 1$ \\
\hline Total & $2767(94,30 \mathrm{p} .100)$ & $166 \quad(5,70$ p. 100$)$ & $16,66: 1$ \\
\hline
\end{tabular}

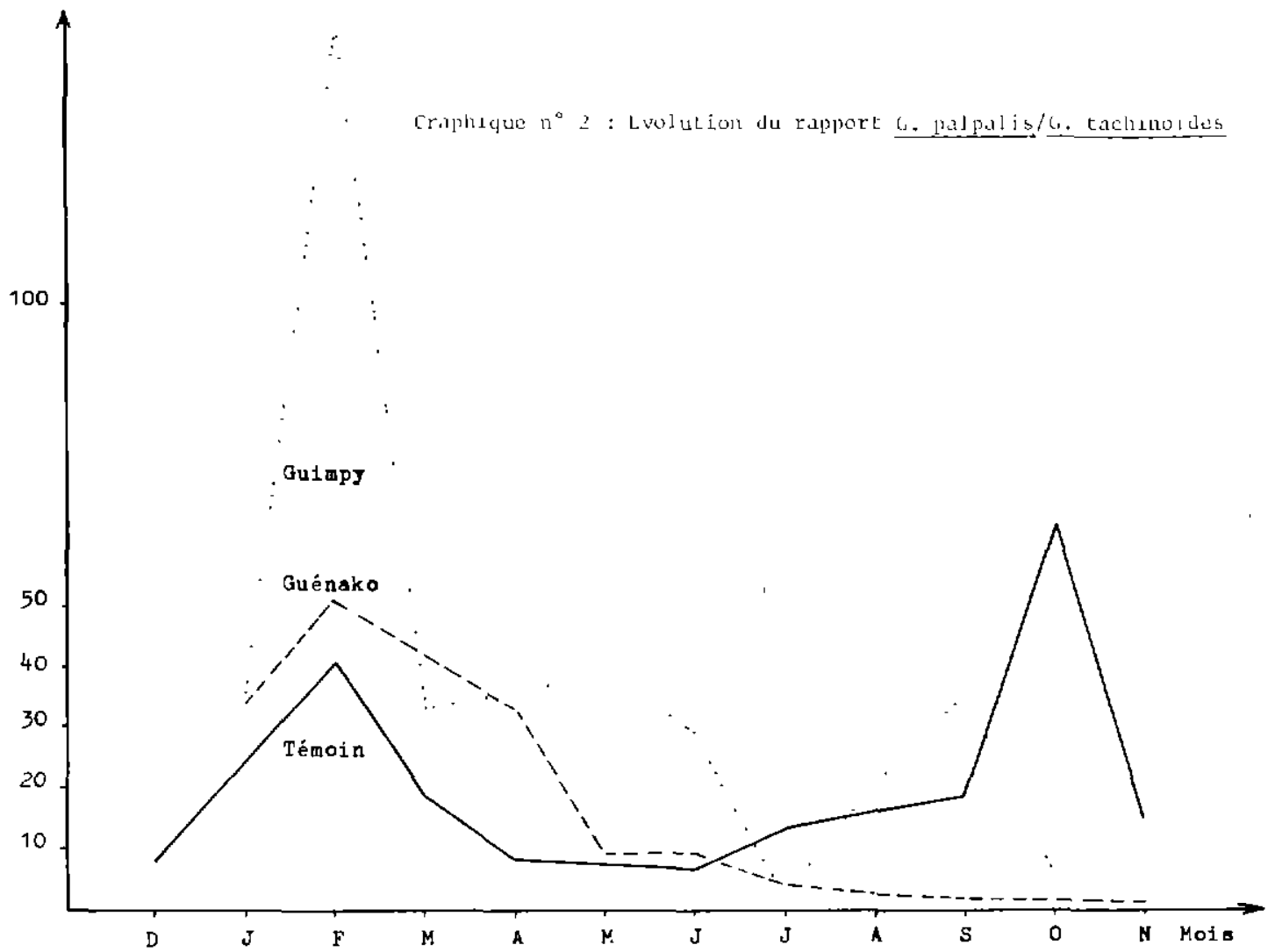

Dans le rapport $G$. palpalis/G. tachinoides, le dénominateur devant être plus élevé qu'il ne l'est du fait des barrières, la baisse de densité de G. p. gambiensis est donc plus importante qu'il n'apparaît et résulte d'un effet des mâles stériles lâchés sur ces gîtes, comme va le montrer l'analyse des résultats suivants.

TABL. $\mathrm{N}^{\circ} \mathrm{V}$-Rëcoltes comparées de G.p.ganibiensi et $G$.tachinoides dans les gites et dans les barrières (glossines marquées).

\begin{tabular}{|l|c|c|}
\hline \multirow{2}{*}{$\begin{array}{l}\text { Gîtes } \\
\text { (20 captures) }\end{array}$} & 959 & 54 \\
\cline { 2 - 3 } & G. paipaizis & G. tachinoides \\
\hline $\begin{array}{l}\text { Barrières } \\
\text { (365 captures) }\end{array}$ & 2604 & 242 \\
\hline Rapport & 2,71 & 4,48 \\
\hline
\end{tabular}

c) Evolution de la densité de population de G. p. gambiensis

Ce critère est certainement le plus évocateur. La comparaison des niveaux de population de l'espèce visée, $G . p$. gambiensis, entre les gîtes expérimentaux et le gîte témoin traduit l'effet des mâles stériles lâchés.

Pour mesurer cette évolution, on a utilisé d'une part l'indice de Lincoln, basé sur des capturesmarquages-recaptures, qui fournit la densité réelle, d'autre part l'abondance relative ou densité apparente.

L'évaluation de la densité d'une population de glossines est toujours difficile et le devient encore plus lorsque les effectifs capturés s'amenuisent, ce qui est le cas ici à la suite du processus 


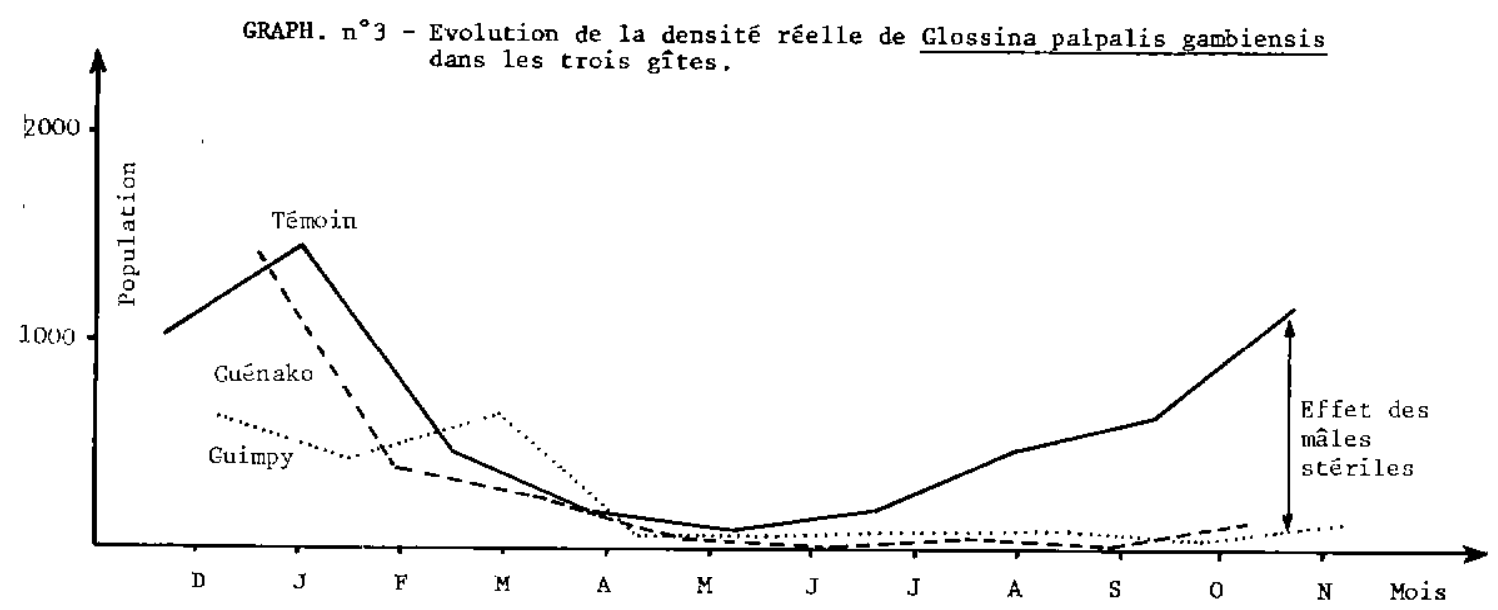

d'éradication engagé. Cependant, les études antérieures (9) ont montré que, sur ce réseau hydrographique, le rapport densité réelle/densité apparente est de 10 environ.

Ce coefficient a donc été utilisé pour obtenir la transformation de la densité apparente à la densité réelle lorsque l'estimation de cette dernière devenait peu fiable du fait des très faibles quantités d'individus capturés.

\section{Densité réelle}

On peut distinguer sur le graphique $\mathrm{n}^{\circ} 3$ 2 parties bien distinctes : décembre à mai et mai à novembre.

- décembre à mai :

Les courbes de densité réelle concernant les populations des 3 gîtes suivent, durant cette période, une évolution très semblable entre-elles et très semblable à celle observée l'année précédant cette expérimentation. Les fortes densités enregistrées en fin des pluies-début de saison sèche baissent régulièrement durant la saison sèche et atteignent un minimum au mois de mai.

- mai à novembre :

A partir de mai, les courbes se dissocient nettement. La densité du témoin va s'accroître à mesure que l'on se rapproche de la saison des pluies pour atteindre au début de la saison sèche suivante le niveau observé 12 mois auparavant.

Par contre, la densité de Guimpy et de Guénako ne subit aucune augmentation et restera jusqu'à novembre au bas niveau qu'elle avait atteint en mai.

\section{Densité apparente}

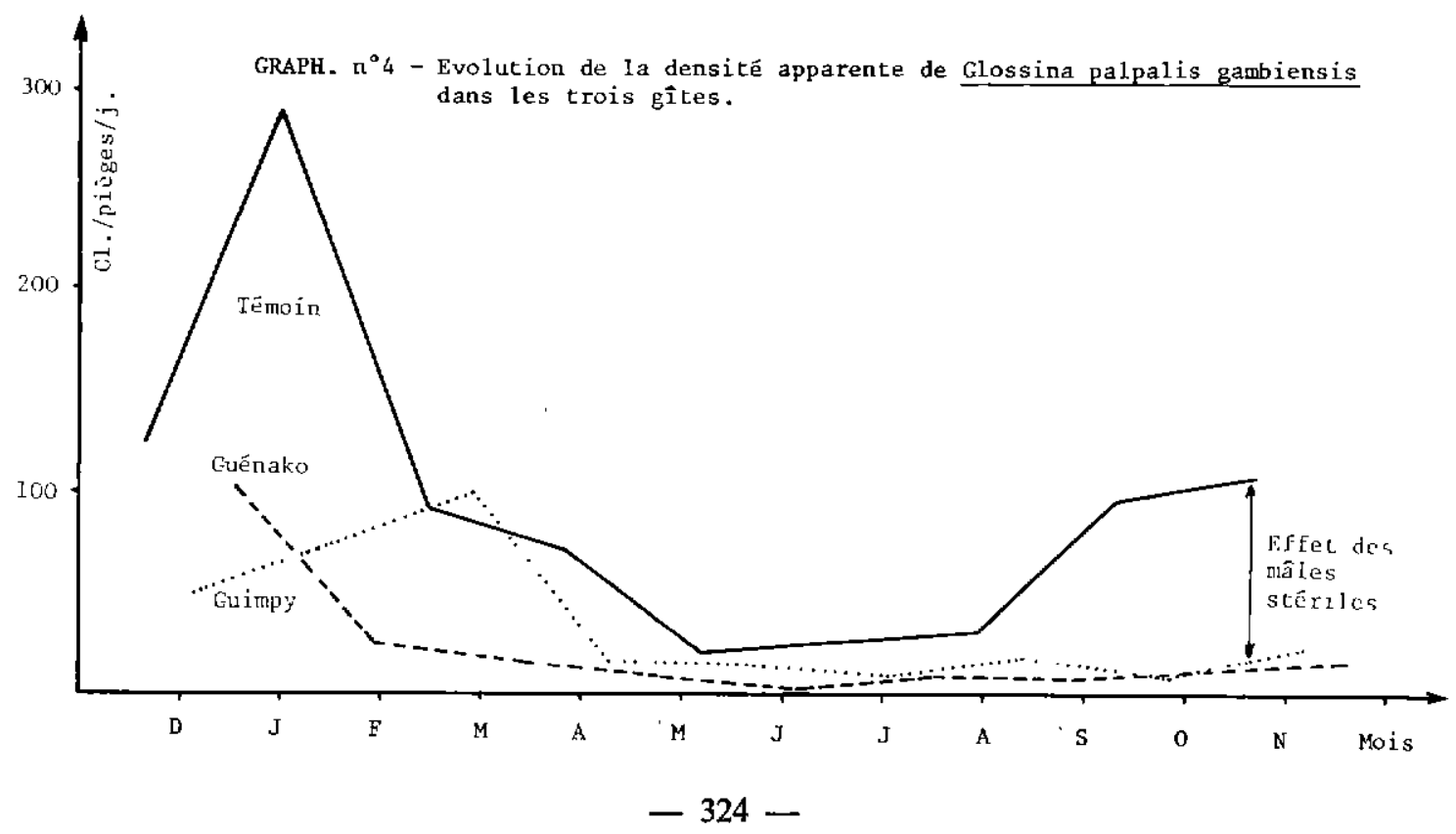


La densité apparente (graphique $n^{\circ} 4$ ) suit une évolution très semblable. Après une décroissance en saison sèche jusqu'au mois de mai sur les 3 gîtes, la densité s'accroît à nouveau sur le témoin tandis qu'elle reste à un bas niveau sur les 2 gîtes d'expérience.

\section{Baisse de densité}

On a donc obtenu une très nette dissociation des courbes de densité apparente 5 mois $1 / 2$ environ après le premier lâcher.

Le tableau VI compare la baisse de densité sur les 3 gîtes entre le moment précédant immédiatement les lâchers et la fin de l'observation, soit à 12 mois d'écart (décembre 1976 à novembre 1977).

Densité apparente et densité réelle donnent des résultats rigoureusement comparables. Le gîte témoin a retrouvé, à 12 mois d'écart, sa densité d'origine, tandis que les 2 gîtes expérimentaux ont perdu 93 p. 100 (Guénako) et 85 p. 100 (Guimpy) de la leur. Ce résultat qui est certainement le plus significatif de l'action des mâles stériles, traduit, de façon indubitable, l'efficacité, sur le terrain, de la lutte autocide contre G. p. gambiensis.

Des facteurs intercurrents ont certainement influencé la densité des gîtes, mais outre que leur action a été faible, elle a agi dans le même sens sur les 3 gîtes.

Parmi eux, l'effet des barrières d'isolement est certainement le plus important.

Il est dû à la capture de glossines qui veulent franchir les barrières $A$ et $B$ (piégeage permanent). Les relevés de capture sur ces dernières (non publié) montrent que cet effet est d'environ 5 p. 100 et qu'il est identique sur la densité des 2 gîtes témoin et Guénako qu'elles séparent.
TABL, $N^{\circ}$ VII -Glossines sauvages marquées sur Tẻmoin et Guénako et recapturées dans les barrières $A$ et $B$.

\begin{tabular}{|c|c|c|}
\hline & Tëroin & Guënako \\
\cline { 2 - 3 } Lâchées & 881 & 155 \\
\hline Recapturées & 48 & 8 \\
\hline Pourcentage & 5,4 & 5,2 \\
\hline
\end{tabular}

Le traitement insecticide, associé au piégeage dans les barrières, ayant un effet au moins égal, l'impact des barrières sur la baisse de population des gîtes est estimé à environ 10 p. 100 et est identique pour témoin et Guénako. Il est certainement un peu plus faible pour Guimpy dont les barrières mitoyennes n'ont subi que des traitements insecticides et pas de piégeage.

Donc, considérant que les effets intercurrents sont pratiquement semblables et assez réduits, l'impact des lâchers de mâles stériles a entraîné la disparition presque totale des glossines sauvages sur les deux tronçons expérimentaux alors que le témoin, après la baisse naturelle de saison sèche, a retrouvé sa densité d'origine.

\section{d) Evolution de la fertilité des femelles sauvages mises en observation}

Tous les trimestres, des lots de femelles récoltées sur chacun des tronçons expérimentaux (161 femelles au total), ont été mis en observation au laboratoire. Chaque femelle a été placée en cage individuelle et sa descendance suivie. L'appareil génital a été systématiquement disséqué après la mort des femelles en observation et la descendance remise dans les gites d'origine, après enregistrement.

TABL. N*VI -Densités comparêes avant et après 12 mois de tâchers de mầies stëriles.

\begin{tabular}{|c|c|c|c|c|}
\hline \multirow{4}{*}{$\begin{array}{l}\text { Densité } \\
\text { rêelle }\end{array}$} & & $\mathrm{T}$ é $\square \circ \mathrm{i} \Omega$ & G u e n a k o & G u i m $p y$ \\
\hline & Inıtiale & 1115 & 1857 & 580 \\
\hline & Finale & I 173 & $\# \quad 130$ & \# 90 \\
\hline & Baisse & $0 \mathrm{p} \cdot 100$ & \#93 p. 100 & $\# 85$ p. 100 \\
\hline \multirow{3}{*}{$\begin{array}{l}\text { Densité } \\
\text { apparente }\end{array}$} & Initiale & 128 & 174 & 79 \\
\hline & Finale & 107 & 13 & 9 \\
\hline & Baisse & $16,40 \mathrm{P} \cdot 100$ & $92,52 \mathrm{p}, 100$ & $88,60 \mathrm{p} \cdot 100$ \\
\hline
\end{tabular}




\section{Femelles à fertilité perturbée}

Ces perturbations peuvent être variables. Ce sont, par ordre d'importance croissante :

- des anomalies de la période précédant la première larviposition;

- des anomalies de la période séparant

2 larvipositions;

- des anomalies ovaro-utérines: blocages, dégénérescences de follicules et d'œufs ;

- la stérilité.

Sur 12 mois d'observation, les pourcentages moyens de fertilité anormale concernant les 3 gites sont respectivement les suivants :

— Guénako : 49,01 p. 100.

- Guimpy : 73,07 p. 100.

— Témoin : 27,58 p. 100.

Il faut noter en particulier qu'au cours du $3^{\mathbf{e}}$ trimestre d'observation, les pourcentages étaient les suivants :

— Guénako : 71,42 p. 100.

- Guimpy : 100,00 p. 100.

一 Témoin : 28,57 p. 100.

\section{Femelles à fertilité supprimée}

Les pourcentages moyens de stérilité totale observée sur les 12 mois d'expérimentation sont les suivants :

- Guénako : 13,72 p. 100.

— Guimpy: 32,69 p. 100.

- Témoin : 0 p. 100.

Cette analyse de la fertilité des femelles récoltées sur le terrain montre nettement que l'impact des mâles stériles est certain. Elle indique également que la baisse très forte de densité enregistrée sur les gîtes d'expérience est bien la conséquence de la lutte autocide engagée. Elle confirme également une infiltration de glossines sauvages sur les gîtes de lâchers, très sensible en saison des pluies, qui n'a pas permis d'obtenir de meilleurs taux de stérilité. Ceux-ci ont en effet augmenté du $1^{\text {ex }}$ au $3^{\text {e }}$ trimestre (début des pluies) puis ont baissé ensuite (saison des pluies). De même, le pourcentage de fertilité anormale sur le témoin $(28,57$ p. 100$)$ est une conséquence d'invasions, réduites mais continues, de mâles stériles.

\section{DISCUSSION}

Les lâchers initiaux ont permis de respecter les taux de mâles stériles fixés, à savoir $3 \not \varnothing: 1 \not$ sur Guénako et $7 \varnothing: 1$ ô sur Guimpy.
L'égalité des quantités lâchées ensuite sur ces 2 gîtes de densité initiale différente (1 800 glossines sur Guénako et 600 glossines sur Guimpy) aboutit à des taux moyens identiques de mâles stériles (\#10:1) qui engendrent en moyenne 49 p. 100 de perturbations de fertilité sur l'un et 73 p. 100 sur l'autre en 12 mois d'expérimentation.

D'après les contrôles effectués au niveau des barrières, l'explication de ce paradoxe est la suivante :

- L'obtention d'un rapport moyen $\varnothing: \hat{o}$ identique sur les 2 gîtes est due :

- d'une part à des échanges à travers la barrière $\mathrm{C}$ de mâles stériles entre Guimpy et Guénako, avec bilan positif pour celle-ci ;

- d'autre part à une élimination de glossines sauvages sur Guénako à la suite de leur piégeage dans les barrières $\mathrm{A}$ et $\mathrm{B}$, ce qui n'est pas le cas pour les glossines de Guimpy.

- A ce taux de mâles stériles identiques sur ces 2 gîtes, la différence des pourcentages de perturbations de la fertilité sur chacun d'eux est due à des infiltrations de glossines sauvages jugées plus fortes sur Guénako que sur Guimpy.

Très laborieusement installées, considérablement renforcées et surveillées, les barrières n'ont pas atteint l'efficacité totale souhaitée. Influant légèrement sur kes densités des gîtes mitoyens et ne remplissant pas de façon absolue leur fonction, les barrières constituent «un mal nécessaire " dont il est très difficile de s'affranchir.

Elles ont révélé, au cours de cette expérimentation, les grandes capacités de vol de G. p. gambiensis qui peut franchir en peu de temps plusieurs kilomètres, à travers un milieu hostile, sans prendre relai (non publié).

La non-extinction de la population sauvage nous semble due à la perméabilité résiduelle de ces barrières, ces faibles infiltrations assurant la pérennité de l'espèce dans les gîtes traités.

Toutefois, au cours de l'année 1978, à la suite de nouvelles mesures de renforcement des barrières qui semblent avoir stoppé toute invasion, les sondages par capture aux pièges sont devenus négatifs, traduisant l'éradication de l'espèce. (Ces derniers résultats qui confirment le succès de l'expérimentation, feront l'objet d'une prochaine publication.) 


\section{CONCLUSION}

Des lâchers inondatifs et ininterrompus de mâles irradiés à 11000 rads ont provoqué une chute de densité nette et importante chez des populations sauvages de $G$. p. gambiensis dont le potentiel de croissance est alors très amoindri, en particulier au moment de la saison des pluies, époque qui leur est pourtant favorable.

Ces performances ont été obtenues en effectuant des lâchers massifs, bihebdomadaires et ininterrompus.

Ces premiers résultats montrent qu'il est nécessaire d'obtenir au départ et de maintenir pendant toute la durée d'intervention un rapport $\not ̛$ : ơ proche de $10: 1$, avec des mâles issus d'un élevage fonctionnant correctement. La dose d'irradiation de 11000 rads a donné satisfaction sur le terrain en accord avec les travaux de laboratoire (16). Les délais de 11 jours et 14 jours entre le traitement insecticide non rémanent et les lâchers de mâles irradiés gagnaient à être raccourcis considérablement.

En effet, des lâchers faisant suite immédiatement à un tel traitement insecticide devraient permettre aux mâles irradiés d'entrer en contact avec une population de densité légèrement plus basse, mais surtout dont la fraction femelle est représentée par des vierges nouvellement écloses, donc très réceptives à l'action des mâles stériles.

L'intégration des 2 méthodes, chimique et biologique, permet d'espérer alors une efficacité accrue dans des délais plus brefs.

\title{
SUMMARY
}

Impact of sterilized male releases on the ratio of two wild fly populations of Glossina palpalis gambiensis in Upper Volta (Black Volta sources)

Males, irradiated with 11000 rads were released for 12 months into two experimental sites consisting of gallery forests, isolated by totally cleared barriers, that fringe the rivers Guimpy and Guenako.

The first releases took place at the beginning of the dry season, 11 and 14 days after a non persistent insecticide application. The ratio of sterile/wild males, initially $3: 1$ and $7: 1$ in these sites reached monthly figures usually higher than 10:1.

The impact of sterile males was estimated by comparison with the control section following different criterions of which the most significant was the dramatic decrease in density of G.p. gambiensts in the experimental sites.

The persistence of a very low residual population in the release sites is essentially due to the insufficient efficiency of the barriers which flies, coming from the outside have been able to cross. Further reinforcement of barriers resulted in the extinction of this population.

\section{RESUMEN}

\author{
Influencia de las sueltas de machos esteriles sobre los niveles \\ de dos poblaciones salvajes de Glossina palpalis gambiensis \\ en Alto-Volta (Fuentes de la Volta Negra)
}

Se soltaron durante 12 meses machos jrradiados con 11000 rads en dos sitios experimentales, constituidos por galerias forestales aisladas por barreras de desmonte, en las orillas de los rios Guimpy y Guenako, fuente de la Volta Negra.

Al principio de la estación seca, se efectuaron las primeras sueltas, 11 y 14 diás después de un tratamıento insecticido no remanente

La relación machos esteriles : machos salvajes, inicialmente de $3: 1$ y $7: 1$ en dichos sitios llegá a valores por mes generalmente superiores a $10: 1$.

Se evaluó la influencia de los machos esteriles por comparación con un sector testigo, según diferentes criterios de los cuales el más significativo es la importante disminución de densidad de G. p. gambiensis en los sitios experimentales.

Una eficacia insuficiente de las barreras es causa esencialmente del mantenimiento de una población residual poco numerosa en los sitios de sueltas. Así glosinas proviniendo del exterior pudieron salvar dichas barreras. Por eso, el refuerzo ulterior de las barreras permitió, en 1978, obtener la extinción total de esta población. 


\section{BIBLIOGRAPHIE}

1. CHALLIER (A.), LAVEISSIÈRE (C.). Un nouveau piège pour la capture des glossines (Glossina : DipteraMuscidae), description et essais sur le terrain. Cah. o. R. S. T. O. M., sér. Ent. méd. Parasit., 1973, 11 (4) : 251-262.

2. CLAIR (M.), CUISANCE (D.), POLITZAR (H.), TAZE (Y.). Début d'expérimentation de lâchers de mâles stériles sur le terrain à Bobo-Dioulasso (HauteVolta). $15^{\mathrm{e}}$ réunion de l'O. U. A./C.S. T. R./ C. S. I. R. C. T., Bandjul (Gambie), 25-30 avril 1977.

3. CLAIR (M.), CUISANCE (D.), POLITZAR (H.), SELLIN (E.), TAZE (Y.). Synthèse des recherches effectuées en 1975-1976 et 1977 sur la lutte génétique contre Glossina palpalis gambiensis Vanderplank, 1949 par lâchers de mâles irradiés. (Bobo-Dioulasso, I. E. M. V. T.-G. T. Z.), Maisons-Alfort, I. E. M. V. T. $1978,43 \mathrm{p}$.

4. CLAIR (M.), POLITZAR (H.), CUISANCE (D.), LAFAYE (A.). Observations sur un essai préliminaire de lâchers de mâles stériles de Glossina palpalis gambiensis (Haute-Volta). Rev. Elev. Méd. vét. Pays trop., 1976, 29 (4) : 241-351.

5. CUISANCE (D.), ITARD (J.). Lâchers de mâles stériles de Glossina tachinoides West. dans un gîte naturel de faible densité (Bas Logone, Cameroun). Rev. Elev. Méd. vét. Pays trop., 1973, 26 (4) : 405-422.

6. CURTIS (C. F.), LANGLEY (P. A.), MEWS (A. R.), OFFORI (E. D.), SOUTHERN (D. 1.), BELL (P. E.). Sex-ratio distortion and semi-sterility in the progeny of irradiated Glossina morsitans, Genet. Res. Camb., 1973, 21 : 153-165.

7. DAME (D. A.), SCHMIDT (C. H.). The sterile male technique against tsetse flies, Glossina spp, Bull. ent. Soc. Am., 1970, 16 (1) : 24-30.

8. EVENS (F.), D'AESELEER (F.). Multiple matings with treated and untreated mâles. Joint F. A. O.l I. A. E. A. Research Coordination Meeting on the
Sterile Insect Technique for Testse Fly Eradication or Control, Bobo-Dioulasso (Upper-Volta), 13-20 novembre 1976.

9. I. E. M. V. T. Rapport annuel 1976, Centre de Recherche sur les Trypanosomiases Animales, BoboDioulasso (Haute-Volta), $82 \mathrm{p}$.

10. I. E. M. V. T. Rapport annuel 1977, Centre de Recherche sur les Trypanosomiases Animales, Bobo-Dioulasso (Haute-Volta), $90 \mathrm{p}$.

11. ITARD (J.). Irradiation "gamma » de mâles de $G, p$. gambiensis. Rapport annuel 1976 du service d'Entomologie de l'I. E. M. V. T. Maisons-Alfort.

12. ITARD (J.). Stérilisation par irradiation gamma de mâles adultes de Glossines. Irradiation à faibles doses (4000 à 6000 rads) de mâles adultes de $G$. tachinoides. $13^{\mathrm{e}}$ réunion C. S. I. R. T. Lagos, 1971. (Publ. $n^{0} 105$ ) p. 325-329.

13. ITARD (J.), CUISANCE (D.), CLAIR (M.), POLITZAR (H.), TAZE (Y.). La lutte génétique contre les mouches tsé-tsé. Cours de formation de spécialistes de la recherche sur les trypanosomiases animales et de la lutte contre ces maladies (Afrique au Sud du Sahara). Maisons-Alfort, septembre 1977.

14. SELLIN (E.), POLITZAR (H.), CUISANCE (D.), CLAIR (M.). L'élevage de Glossina palpalis gambiensis Vanderplank 1949 (Diptera-Muscidae) à BoboDioulasso (Haute-Volta). Rev. Elev. Méd. vét. Pays trop., 1977,30 (1) : 41-49.

15. SOUTHWOOD (T. R. E.). Ecological methods with particular reference to the study of insect populations. London, Chapman and Hall, 1976. 391 p.

16. TAZE (Y.), CUISANCE (D.), POLITZAR (H.), CLAIR (M.), SELLIN (E.). Essai de détermination de la dose optimale d'irradiation des mâles de Glossina palpalis gambiensis (Vanderplank 1949). Rev. Elev. Méd. vét. Pays trop., 1977, 30 (3) : 269-279. 